\title{
Possible role for arginine-containing toothpastes in managing dentine hypersensitivity
}

\section{Abstracted from}

\section{Sharif MO, Iram S, Brunton PA.}

Effectiveness of arginine-containing toothpastes in treating dentine hypersensitivity: A systematic review. J Dent 2013; 41: 483-492.

Address for correspondence: Mohammad O. Sharif, School of Dentistry, University of Manchester, Coupland, 3, Oxford Road, Manchester M13 9PL, UK. E-mail: mohammad.sharif@manchester.ac.uk

\section{Question: Are arginine-containing toothpastes effective for reducing dentine hypersensitivity?}

Data sources The Cochrane Central Register of Controlled Trials (CENTRAL), Medline, Embase and LILACS databases were searched. Reference lists of eligible studies and systematic reviews were crosschecked to identify additional studies.

Study selection Randomised and controlled clinical trials conducted in adults with at least two hypersensitive teeth confirmed by evaporative stimulus or tactile hypersensitivity assessment comparing argininecontaining desensitising toothpastes to non-arginine-containing control toothpastes were included.

Data extraction and synthesis Data abstraction and risk of bias assessment were conducted independently by two reviewers and a qualitative summary presented.

Results Two randomised trials met the criteria; both showed a positive effect on dentine hypersensitivity in response to tactile and air-blast stimuli. No subjective measures of sensitivity were reported.

Conclusions Data identified indicate a potential role for argininecontaining toothpastes in managing dentine hypersensitivity. However, this conclusion is based on small sample sizes and the studies identified did not follow patients up in the medium to long term. The authors recommend that there is a need for well-designed RCTs to be conducted prior to any definitive recommendations being made.

\section{Commentary}

Dentine hypersensitivity is often described as an immediate pain which arises from exposed dentine when stimulus is applied. ${ }^{1}$ Limited evidence is available on individual agents and their success in providing patients with long term relief from the pain hypersensitive teeth can bring. A systematic review of the literature by Po-Yen Lin et al. in 2012 suggested that most dentine hypersensitivity treatments do provide some benefit for patients. ${ }^{2}$ Another recent study by Boneta et al. which compared the use of arginine- containing toothpaste against potassium nitrate toothpaste and a standard fluoride toothpaste suggested better outcomes for the arginine-containing toothpaste intervention group at eight weeks. ${ }^{3}$

This systematic review examines evidence of the effectiveness of arginine-containing toothpastes in reducing dentine hypersensitivity. The reviewers provide a clear description of the methods used. They searched four relevant databases (Medline, CENTRAL, Embase and LILACS) for randomised control and controlled clinical trials as well as cross-checking the reference lists of identified studies and contacting experts on this topic to identify areas of grey literature. The population group to be studied was restricted to adults who had at least two teeth affected by hypersensitivity. Changes in pain/sensitivity reported following a tactile or blast stimulus to the teeth was considered as the primary outcome. In addition subjective reports of immediate or delayed relief from sensitivity were included as a secondary outcome measure.

Sixty-nine potential studies were identified in the initial search. After screening, two randomised control trials remained which met the inclusion criteria. These trials were both carried out by the same group of authors and included 243 participants in total. Risk of bias assessment was carried out by the reviewers, with both studies deemed to have an unclear risk of bias. A brief narrative review of each of the two studies describes the primary outcomes alongside tables of the summary characteristics and intervention effects. One of the studies found a significantly greater reduction in dentine hypersensitivity following stimulus to the teeth when using a test toothpaste containing $8.0 \%$ arginine compared to a negative control toothpaste at 2, 4 and 8 weeks. The second study looked at changes in hypersensitivity after three days of intervention with one of three toothpastes; toothpaste containing arginine, toothpaste containing arginine and a whitening agent and a control toothpaste. This study also found a significant reduction in dentine hypersensitivity between arginine-containing toothpaste and the control. Given that only two studies met the 
inclusion criteria neither meta-analysis nor subgroup or sensitivity analysis were performed.

The reviewers found no evidence to report on the secondary outcome of subjective measurement of sensitivity. Challenges remain in how to reliably measure dentine hypersensitivity. Studies such those included in this review use standardised pain measurement scales and tactile/air blast stimuli, however, a level of patient subjectivity still remains.

There have been very few high quality studies which look at a direct comparison between arginine-containing toothpastes and those which don't. The studies included in this systematic review suggest an improvement in hypersensitivity with the use of an arginine-containing toothpaste. However, given the limited number of studies identified for review and the short follow-up period we cannot reliably confirm that long-term use of an arginine-containing toothpaste will improve the symptoms of dentine hypersensitivity. The reviewers also acknowledge that the potential risk of bias mean these results should be interpreted with caution. This is an area which warrants medium to long-term study and there remains a need for well conducted studies to assess the effectiveness of desensitising toothpastes.

\section{Susan J Carson}

Dundee Dental School, Park Place, Dundee, Scotland, UK.

1. Holland GR, Narhi MN, Addy M, Gangarosa L, Orchardson R. Guidelines for the design and conduct of clinical trials on dentine hypersensitivity. J Clin Periodontol 1997; 24: 808-813.

2. Lin PY, Cheng YW, Chu CY, Chien KL, Lin CP, Tu YK. In-office treatment for dentin hypersensitivity: a systematic review and network meta-analysis. / Clin Periodontol 2013; 40: 53-64.

3. Elias Boneta AR, Ramirez $\mathrm{K}, \mathrm{Naboa}$ I, et al. Efficacy in reducing dentine hypersensitivity of a regimen using a toothpaste containing $8 \%$ arginine and calcium carbonate, a mouthwash containing $0.8 \%$ arginine, pyrophosphate and PVM/MA copolymer and a toothbrush compared to potassium and negative control regimens: an eightweek randomized clinical trial. J Dent 2013; 41: Suppl 1:S42-49. doi: 10.1016/j. jdent.2012.11.011. Epub 2013 Feb 1.

Evidence-Based Dentistry (2013) 14, 44-45. doi:10.1038/sj.ebd.6400930 